\title{
Ostrowski type fractional integral inequalities for MT-convex functions
}

\author{
Wenjun Liu
}




\title{
OSTROWSKI TYPE FRACTIONAL INTEGRAL INEQUALITIES FOR MT-CONVEX FUNCTIONS
}

\author{
WENJUN LIU \\ Received 12 February, 2014
}

\begin{abstract}
Some inequalities of Ostrowski type for MT-convex functions via fractional integrals are obtained. These results not only generalize those of [25], but also provide new estimates on these types of Ostrowski inequalities for fractional integrals.
\end{abstract}

2010 Mathematics Subject Classification: 26A33; 26A51; 26D07; 26D10; 26D15

Keywords: Ostrowski type inequality, MT-convex function, fractional integral

\section{INTRODUCTION}

The following result is known in the literature as the Ostrowski inequality (see [17, page 468] or [18]), which gives an upper bound for the approximation of the integral average $\frac{1}{b-a} \int_{a}^{b} f(t) d t$ by the value $f(x)$ at point $x \in[a, b]$.

Theorem 1. Let $f: I \rightarrow \mathbb{R}$, where $I \subseteq \mathbb{R}$ is an interval, be a mapping differentiable in the interior $I^{\circ}$ of $I$, and let $a, b \in I^{\circ}$ with $a<b$. If $\left|f^{\prime}(x)\right| \leq M$ for all $x \in[a, b]$, then

$$
\left|f(x)-\frac{1}{b-a} \int_{a}^{b} f(t) d t\right| \leq M(b-a)\left[\frac{1}{4}+\frac{\left(x-\frac{a+b}{2}\right)^{2}}{(b-a)^{2}}\right], \quad \forall x \in[a, b] .
$$

In recent years, various generalizations, extensions and variants of such inequalities have been obtained (see $[1,4,5,8,10-15,20,24]$ and the references cited therein). In [23] (see also [25, 26]), Tunç and Yidirim defined the following so-called MTconvex function:

Definition 1. A function: $I \subseteq \mathbb{R} \rightarrow \mathbb{R}$ is said to belong to the class of $\operatorname{MT}(I)$, if it is nonnegative and for all $x, y \in I$ and $t \in(0,1)$ satisfies the following inequality:

$$
f(t x+(1-t) y) \leq \frac{\sqrt{t}}{2 \sqrt{1-t}} f(x)+\frac{\sqrt{1-t}}{2 \sqrt{t}} f(y) .
$$

(c) 2015 Miskolc University Press 
In [25], Tunç derived some inequalities of Ostrowski type for MT-convex functions.

Theorem 2. Let $f:[a, b] \subset[0, \infty) \rightarrow \mathbb{R}$ be a differentiable mapping on $(a, b)$ with $a<b$ such that $f^{\prime} \in L_{1}[a, b]$. If $\left|f^{\prime}\right|$ is MT-convex on $[a, b]$ and $\left|f^{\prime}(x)\right| \leq M$, $x \in[a, b]$, then we have

$$
\left|f(x)-\frac{1}{b-a} \int_{a}^{b} f(t) d t\right| \leq \frac{M \pi\left[(x-a)^{2}+(b-x)^{2}\right]}{4(b-a)}
$$

for each $x \in[a, b]$.

Theorem 3. Let $f:[a, b] \subset[0, \infty) \rightarrow \mathbb{R}$ be a differentiable mapping on $(a, b)$ with $a<b$ such that $f^{\prime} \in L_{1}[a, b]$. If $\left|f^{\prime}\right|^{q}$ is MT-convex on $[a, b], q>1, p^{-1}+q^{-1}=1$ and $\left|f^{\prime}(x)\right| \leq M, x \in[a, b]$, then we have

$$
\left|f(x)-\frac{1}{b-a} \int_{a}^{b} f(t) d t\right| \leq \frac{M}{(1+p)^{1 / p}}\left(\frac{\pi}{2}\right)^{\frac{1}{q}} \frac{(x-a)^{2}+(b-x)^{2}}{(b-a)}
$$

for each $x \in[a, b]$.

Theorem 4. Let $f:[a, b] \subset[0, \infty) \rightarrow \mathbb{R}$ be a differentiable mapping on $(a, b)$ with $a<b$ such that $f^{\prime} \in L_{1}[a, b]$. If $\left|f^{\prime}\right|^{q}$ is MT-convex on $[a, b], q \geq 1$ and $\left|f^{\prime}(x)\right| \leq M$, $x \in[a, b]$, then we have

$$
\left|f(x)-\frac{1}{b-a} \int_{a}^{b} f(t) d t\right| \leq M\left(\frac{1}{2}\right)^{1+\frac{1}{q}} \pi^{\frac{1}{q}} \frac{(x-a)^{2}+(b-x)^{2}}{(b-a)}
$$

for each $x \in[a, b]$.

Fractional calculus $[7,16,19]$ was introduced at the end of the nineteenth century by Liouville and Riemann, the subject of which has become a rapidly growing area and has found applications in diverse fields ranging from physical sciences and engineering to biological sciences and economics. We recall definitions and preliminary facts of fractional calculus theory which will be used in this paper.

Definition 2. Let $f \in L_{1}[a, b]$. The Riemann-Liouville integrals $J_{a+}^{\alpha} f$ and $J_{b-}^{\alpha} f$ of order $\alpha>0$ with $a \geq 0$ are defined by

$$
J_{a+}^{\alpha} f(x)=\frac{1}{\Gamma(\alpha)} \int_{a}^{x}(x-t)^{\alpha-1} f(t) d t, x>a
$$

and

$$
J_{b-}^{\alpha} f(x)=\frac{1}{\Gamma(\alpha)} \int_{x}^{b}(t-x)^{\alpha-1} f(t) d t, \quad x<b,
$$

respectively, where $\Gamma(\alpha)=\int_{0}^{\infty} e^{-u} u^{\alpha-1} d u$. Here, $J_{a+}^{0} f(x)=J_{b-}^{0} f(x)=f(x)$. In the case of $\alpha=1$, the fractional integral reduces to the classical integral. 
Due to the wide application of fractional integrals, some authors extended to study fractional integral inequalities, we refer the reader to the papers $[2,3,6,9,21]$ and the reference cited therein.

Motivated by these results, in the present paper, we establish some Ostrowski type inequalities for MT-convex functions via Riemann-Liouville fractional integrals. So, new estimates on these types of Ostrowski inequalities via fractional integrals are provided and the results of [25] are generalized.

\section{OSTROWSKI TYPE FRACTIONAL INTEGRAL INEQUALITIES FOR MT-CONVEX FUNCTIONS}

In this section, we apply the following fractional integral identity from Set [22] to derive some new Ostrowski type fractional integral inequalities for MT-convex functions.

Lemma 1. Let $f:[a, b] \rightarrow \mathbb{R}$ be a differentiable mapping on $(a, b)$ with $a<b$. If $f^{\prime} \in L_{1}[a, b]$, then for all $x \in[a, b]$ and $\alpha>0$, one has

$$
\begin{aligned}
& \frac{(x-a)^{\alpha}+(b-x)^{\alpha}}{b-a} f(x)-\frac{\Gamma(\alpha+1)}{b-a}\left[J_{x-}^{\alpha} f(a)+J_{x+}^{\alpha} f(b)\right] \\
& =\frac{(x-a)^{\alpha+1}}{b-a} \int_{0}^{1} t^{\alpha} f^{\prime}(t x+(1-t) a) d t-\frac{(b-x)^{\alpha+1}}{b-a} \int_{0}^{1} t^{\alpha} f^{\prime}(t x+(1-t) b) d t .
\end{aligned}
$$

Using this lemma, we can obtain the following Ostrowski type fractional integral inequalities for MT-convex functions.

Theorem 5. Let $f:[a, b] \subset[0, \infty) \rightarrow \mathbb{R}$ be a differentiable mapping on $(a, b)$ with $a<b$ such that $f^{\prime} \in L_{1}[a, b]$. If $\left|f^{\prime}\right|$ is MT-convex on $[a, b]$ and $\left|f^{\prime}(x)\right| \leq M$, $x \in[a, b]$, then the following inequalities for fractional integrals with $\alpha>0$ and $x \in[a, b]$ hold:

$$
\begin{aligned}
& \left|\frac{(x-a)^{\alpha}+(b-x)^{\alpha}}{b-a} f(x)-\frac{\Gamma(\alpha+1)}{b-a}\left[J_{x-}^{\alpha} f(a)+J_{x+}^{\alpha} f(b)\right]\right| \\
& \leq M \frac{\Gamma\left(\alpha+\frac{1}{2}\right) \Gamma\left(\frac{1}{2}\right)}{2 \Gamma(\alpha+1)} \frac{(x-a)^{\alpha+1}+(b-x)^{\alpha+1}}{b-a} .
\end{aligned}
$$

Proof. From (2.1) and since $\left|f^{\prime}\right|$ is MT-convex, we have

$$
\begin{aligned}
& \left|\frac{(x-a)^{\alpha}+(b-x)^{\alpha}}{b-a} f(x)-\frac{\Gamma(\alpha+1)}{b-a}\left[J_{x-}^{\alpha} f(a)+J_{x+}^{\alpha} f(b)\right]\right| \\
& \leq \frac{(x-a)^{\alpha+1}}{b-a} \int_{0}^{1} t^{\alpha}\left|f^{\prime}(t x+(1-t) a)\right| d t \\
& \quad+\frac{(b-x)^{\alpha+1}}{b-a} \int_{0}^{1} t^{\alpha}\left|f^{\prime}(t x+(1-t) b)\right| d t
\end{aligned}
$$




$$
\begin{aligned}
\leq & \frac{(x-a)^{\alpha+1}}{b-a} \int_{0}^{1}\left[t^{\alpha} \frac{\sqrt{t}}{2 \sqrt{1-t}}\left|f^{\prime}(x)\right|+t^{\alpha} \frac{\sqrt{1-t}}{2 \sqrt{t}}\left|f^{\prime}(a)\right|\right] d t \\
& +\frac{(b-x)^{\alpha+1}}{b-a} \int_{0}^{1}\left[t^{\alpha} \frac{\sqrt{t}}{2 \sqrt{1-t}}\left|f^{\prime}(x)\right|+t^{\alpha} \frac{\sqrt{1-t}}{2 \sqrt{t}}\left|f^{\prime}(b)\right|\right] d t \\
\leq & \frac{M(x-a)^{\alpha+1}}{b-a} \int_{0}^{1}\left[t^{\alpha} \frac{\sqrt{t}}{2 \sqrt{1-t}}+t^{\alpha} \frac{\sqrt{1-t}}{2 \sqrt{t}}\right] d t \\
& +\frac{M(b-x)^{\alpha+1}}{b-a} \int_{0}^{1}\left[t^{\alpha} \frac{\sqrt{t}}{2 \sqrt{1-t}}+t^{\alpha} \frac{\sqrt{1-t}}{2 \sqrt{t}}\right] d t \\
= & \frac{M\left[(x-a)^{\alpha+1}+(b-x)^{\alpha+1}\right]}{2(b-a)} \int_{0}^{1}\left[t^{\alpha+\frac{1}{2}}(1-t)^{-1 / 2}+t^{\alpha-\frac{1}{2}}(1-t)^{1 / 2}\right] \mathrm{d} t \\
= & \frac{M\left[(x-a)^{\alpha+1}+(b-x)^{\alpha+1}\right]}{2(b-a)}\left[\beta\left(\alpha+\frac{3}{2}, \frac{1}{2}\right)+\beta\left(\alpha+\frac{1}{2}, \frac{3}{2}\right)\right] \\
= & \frac{M\left[(x-a)^{\alpha+1}+(b-x)^{\alpha+1}\right]}{2(b-a)} \frac{\Gamma\left(\alpha+\frac{1}{2}\right) \Gamma\left(\frac{1}{2}\right)}{\Gamma(\alpha+1)},
\end{aligned}
$$

where we have used the Beta function of Euler type, which is defined as

$$
\beta(x, y)=\int_{0}^{1} t^{x-1}(1-t)^{y-1} d t=\frac{\Gamma(x) \Gamma(y)}{\Gamma(x+y)}, \quad \forall x, y>0 .
$$

The proof is completed.

Remark 1. In Theorem 5, if we choose $\alpha=1$, we get the inequality in Theorem 2 .

Theorem 6. Let $f:[a, b] \subset[0, \infty) \rightarrow \mathbb{R}$ be a differentiable mapping on $(a, b)$ with $a<b$ such that $f^{\prime} \in L_{1}[a, b]$. If $\left|f^{\prime}\right|^{q}$ is MT-convex on $[a, b], q>1, p^{-1}+q^{-1}=1$ and $\left|f^{\prime}(x)\right| \leq M, x \in[a, b]$, then the following inequalities for fractional integrals with $\alpha>0$ and $x \in[a, b]$ hold:

$$
\begin{aligned}
& \left|\frac{(x-a)^{\alpha}+(b-x)^{\alpha}}{b-a} f(x)-\frac{\Gamma(\alpha+1)}{b-a}\left[J_{x-}^{\alpha} f(a)+J_{x+}^{\alpha} f(b)\right]\right| \\
& \leq \frac{M}{(1+p \alpha)^{1 / p}}\left(\frac{\pi}{2}\right)^{\frac{1}{q}} \frac{(x-a)^{\alpha+1}+(b-x)^{\alpha+1}}{b-a} .
\end{aligned}
$$

Proof. From Lemma 1 and using the well-known Hölder's inequality, we have

$$
\begin{aligned}
& \left|\frac{(x-a)^{\alpha}+(b-x)^{\alpha}}{b-a} f(x)-\frac{\Gamma(\alpha+1)}{b-a}\left[J_{x-}^{\alpha} f(a)+J_{x+}^{\alpha} f(b)\right]\right| \\
& \leq \frac{(x-a)^{\alpha+1}}{b-a} \int_{0}^{1} t^{\alpha}\left|f^{\prime}(t x+(1-t) a)\right| d t
\end{aligned}
$$




$$
\begin{aligned}
& +\frac{(b-x)^{\alpha+1}}{b-a} \int_{0}^{1} t^{\alpha}\left|f^{\prime}(t x+(1-t) b)\right| d t \\
\leq & \frac{(x-a)^{\alpha+1}}{b-a}\left(\int_{0}^{1} t^{p \alpha} d t\right)^{\frac{1}{p}}\left(\int_{0}^{1}\left|f^{\prime}(t x+(1-t) a)\right|^{q} d t\right)^{\frac{1}{q}} \\
& +\frac{(b-x)^{\alpha+1}}{b-a}\left(\int_{0}^{1} t^{p \alpha} d t\right)^{\frac{1}{p}}\left(\int_{0}^{1}\left|f^{\prime}(t x+(1-t) b)\right|^{q} d t\right)^{\frac{1}{q}} .
\end{aligned}
$$

Since $\left|f^{\prime}\right|^{q}$ is MT-convex and $\left|f^{\prime}(x)\right| \leq M$, we get

$$
\begin{aligned}
\int_{0}^{1}\left|f^{\prime}(t x+(1-t) a)\right|^{q} d t & \leq \int_{0}^{1}\left[\frac{\sqrt{t}}{2 \sqrt{1-t}}\left|f^{\prime}(x)\right|^{q}+\frac{\sqrt{1-t}}{2 \sqrt{t}}\left|f^{\prime}(a)\right|^{q}\right] d t \\
& \leq M^{q} \int_{0}^{1}\left[\frac{\sqrt{t}}{2 \sqrt{1-t}}+\frac{\sqrt{1-t}}{2 \sqrt{t}}\right] d t=\frac{\pi}{2} M^{q}
\end{aligned}
$$

and similarly

$$
\int_{0}^{1}\left|f^{\prime}(t x+(1-t) b)\right|^{q} d t \leq \frac{\pi}{2} M^{q}
$$

By simple computation, we have

$$
\int_{0}^{1} t^{p \alpha} d t=\frac{1}{p \alpha+1}
$$

Using these results, we complete the proof of (2.3).

Remark 2. In Theorem 6, if we choose $\alpha=1$, we get the inequality in Theorem 3 .

Theorem 7. Let $f:[a, b] \subset[0, \infty) \rightarrow \mathbb{R}$ be a differentiable mapping on $(a, b)$ with $a<b$ such that $f^{\prime} \in L_{1}[a, b]$. If $\left|f^{\prime}\right|^{q}$ is MT-convex on $[a, b], q \geq 1$ and $\left|f^{\prime}(x)\right| \leq M$, $x \in[a, b]$, then the following inequalities for fractional integrals with $\alpha>0$ and $x \in[a, b]$ hold:

$$
\begin{aligned}
& \left|\frac{(x-a)^{\alpha}+(b-x)^{\alpha}}{b-a} f(x)-\frac{\Gamma(\alpha+1)}{b-a}\left[J_{x-}^{\alpha} f(a)+J_{x+}^{\alpha} f(b)\right]\right| \\
& \leq \frac{M}{(1+\alpha)^{1-\frac{1}{q}}}\left(\frac{\Gamma\left(\alpha+\frac{1}{2}\right) \Gamma\left(\frac{1}{2}\right)}{2 \Gamma(\alpha+1)}\right)^{\frac{1}{q}} \frac{(x-a)^{\alpha+1}+(b-x)^{\alpha+1}}{b-a} .
\end{aligned}
$$

Proof. From Lemma 1 and using the well-known power mean inequality, we have

$$
\begin{aligned}
& \left|\frac{(x-a)^{\alpha}+(b-x)^{\alpha}}{b-a} f(x)-\frac{\Gamma(\alpha+1)}{b-a}\left[J_{x-}^{\alpha} f(a)+J_{x+}^{\alpha} f(b)\right]\right| \\
& \leq \frac{(x-a)^{\alpha+1}}{b-a} \int_{0}^{1} t^{\alpha}\left|f^{\prime}(t x+(1-t) a)\right| d t
\end{aligned}
$$




$$
\begin{aligned}
& +\frac{(b-x)^{\alpha+1}}{b-a} \int_{0}^{1} t^{\alpha}\left|f^{\prime}(t x+(1-t) b)\right| d t \\
\leq & \frac{(x-a)^{\alpha+1}}{b-a}\left(\int_{0}^{1} t^{\alpha} d t\right)^{1-\frac{1}{q}}\left(\int_{0}^{1} t^{\alpha}\left|f^{\prime}(t x+(1-t) a)\right|^{q} d t\right)^{\frac{1}{q}} \\
& +\frac{(b-x)^{\alpha+1}}{b-a}\left(\int_{0}^{1} t^{\alpha} d t\right)^{1-\frac{1}{q}}\left(\int_{0}^{1} t^{\alpha}\left|f^{\prime}(t x+(1-t) b)\right|^{q} d t\right)^{\frac{1}{q}} .
\end{aligned}
$$

Since $\left|f^{\prime}\right|^{q}$ is MT-convex on $[a, b]$ and $\left|f^{\prime}(x)\right| \leq M$, we get

$$
\begin{aligned}
& \int_{0}^{1} t^{\alpha}\left|f^{\prime}(t x+(1-t) a)\right|^{q} d t \\
& \leq \int_{0}^{1}\left[t^{\alpha} \frac{\sqrt{t}}{2 \sqrt{1-t}}\left|f^{\prime}(x)\right|^{q}+t^{\alpha} \frac{\sqrt{1-t}}{2 \sqrt{t}}\left|f^{\prime}(a)\right|^{q}\right] d t \\
& \leq M^{q} \int_{0}^{1}\left[t^{\alpha} \frac{\sqrt{t}}{2 \sqrt{1-t}}+t^{\alpha} \frac{\sqrt{1-t}}{2 \sqrt{t}}\right] d t=\frac{\Gamma\left(\alpha+\frac{1}{2}\right) \Gamma\left(\frac{1}{2}\right)}{2 \Gamma(\alpha+1)} M^{q}
\end{aligned}
$$

and similarly

$$
\int_{0}^{1} t^{\alpha}\left|f^{\prime}(t x+(1-t) b)\right|^{q} d t \leq \frac{\Gamma\left(\alpha+\frac{1}{2}\right) \Gamma\left(\frac{1}{2}\right)}{2 \Gamma(\alpha+1)} M^{q} .
$$

Using these inequalities, we complete the proof of (2.4).

Remark 3. In Theorem 7, if we choose $\alpha=1$, we get the inequality in Theorem 4 .

\section{ACKNOWLEDGEMENTS}

This work was partly supported by the National Natural Science Foundation of China (Grant No. 11301277), the Qing Lan Project of Jiangsu Province, and the Training Abroad Project of Outstanding Young and Middle-Aged University Teachers and Presidents.

\section{REFERENCES}

[1] M. Alomari, M. Darus, S. S. Dragomir, and P. Cerone, "Ostrowski type inequalities for functions whose derivatives are $s$-convex in the second sense," Appl. Math. Lett., vol. 23, no. 9, pp. 10711076, 2010.

[2] G. Anastassiou, M. R. Hooshmandasl, A. Ghasemi, and F. Moftakharzadeh, "Montgomery identities for fractional integrals and related fractional inequalities," JIPAM. J. Inequal. Pure Appl. Math., vol. 10, no. 4, pp. Article 97, 6, 2009.

[3] Z. Dahmani, "New inequalities in fractional integrals," Int. J. Nonlinear Sci., vol. 9, no. 4, pp. 493-497, 2010.

[4] S. S. Dragomir, "The Ostrowski integral inequality for mappings of bounded variation," Bull. Austral. Math. Soc., vol. 60, no. 3, pp. 495-508, 1999. 
[5] S. S. Dragomir, "The Ostrowski's integral inequality for Lipschitzian mappings and applications," Comput. Math. Appl., vol. 38, no. 11-12, pp. 33-37, 1999.

[6] B. Dyda, "Fractional Hardy inequality with a remainder term," Colloq. Math., vol. 122, no. 1, pp. 59-67, 2011.

[7] R. Gorenflo and F. Mainardi, "Fractional calculus: integral and differential equations of fractional order," in Fractals and fractional calculus in continuum mechanics (Udine, 1996), ser. CISM Courses and Lectures. Springer, Vienna, 1997, vol. 378, pp. 223-276.

[8] W. Liu, "New integral inequalities via $(\alpha, m)$-convexity and quasi-convexity," Hacet. J. Math. Stat., vol. 42, no. 3, pp. 289-297, 2013.

[9] W. Liu, "Some Ostrowski type inequalities via Riemann-Liouville fractional integrals for $h$ convex functions," J. Comput. Anal. Appl., vol. 16, no. 5, pp. 998-1004, 2014.

[10] W. Liu and X. Gao, "Approximating the finite Hilbert transform via a companion of Ostrowski's inequality for function of bounded variation and applications," Appl. Math. Comput., vol. 247, pp. 373-385, 2014.

[11] W. Liu and Q.-A. Ngô, "A generalization of Ostrowski inequality on time scales for $k$ points," Appl. Math. Comput., vol. 203, no. 2, pp. 754-760, 2008.

[12] W. Liu, Q. A. Ngô, and W. Chen, "On new Ostrowski type inequalities for double integrals on time scales," Dynam. Systems Appl., vol. 19, no. 1, pp. 189-198, 2010.

[13] W. Liu, Q. A. Ngô, and W. Chen, "Ostrowski type inequalities on time scales for double integrals," Acta Appl. Math., vol. 110, no. 1, pp. 477-497, 2010.

[14] Z. Liu, "Some companions of an Ostrowski type inequality and applications," JIPAM. J. Inequal. Pure Appl. Math., vol. 10, no. 2, pp. Article 52, 12, 2009.

[15] Z. Lü, "On sharp inequalities of Simpson type and Ostrowski type in two independent variables," Comput. Math. Appl., vol. 56, no. 8, pp. 2043-2047, 2008.

[16] K. S. Miller and B. Ross, An introduction to the fractional calculus and fractional differential equations, ser. A Wiley-Interscience Publication. John Wiley \& Sons, Inc., New York, 1993.

[17] D. S. Mitrinović, J. E. Pečarić, and A. M. Fink, Inequalities involving functions and their integrals and derivatives, ser. Mathematics and its Applications (East European Series). Kluwer Academic Publishers Group, Dordrecht, 1991, vol. 53.

[18] A. Ostrowski, "Über die Absolutabweichung einer differentiierbaren Funktion von ihrem Integralmittelwert," Comment. Math. Helv., vol. 10, no. 1, pp. 226-227, 1937.

[19] I. Podlubny, Fractional differential equations, ser. Mathematics in Science and Engineering. Academic Press, Inc., San Diego, CA, 1999, vol. 198, an introduction to fractional derivatives, fractional differential equations, to methods of their solution and some of their applications.

[20] M. Z. Sarikaya, "On the Ostrowski type integral inequality," Acta Math. Univ. Comenian. (N.S.), vol. 79, no. 1, pp. 129-134, 2010.

[21] M. Z. Sarikaya and H. Ogunmez, "On new inequalities via Riemann-Liouville fractional integration," Abstr. Appl. Anal., pp. Art. ID 428 983, 10, 2012.

[22] E. Set, "New inequalities of Ostrowski type for mappings whose derivatives are $s$-convex in the second sense via fractional integrals," Comput. Math. Appl., vol. 63, no. 7, pp. 1147-1154, 2012.

[23] M. Tunç, "On $m t$-convexity," arXiv:1205.5453v1 [math.CA].

[24] M. Tunç, "On some integral inequalities via $h$-convexity," Miskolc Math. Notes, vol. 14, no. 3, pp. 1041-1057, 2013.

[25] M. Tunç, "Ostrowski type inequalities for functions whose derivatives are $M T$-convex," J. Comput. Anal. Appl., vol. 17, no. 4, pp. 691-696, 2014.

[26] M. Tunç, Y. Subas, and I. Karabayir, "On some hadamard type inequalities for mt-convex functions,” Int. J. Open Probl. Comput. Sci. Math., vol. 6, no. 2, pp. 102-113, 2013. 
Author's address

Wenjun Liu

College of Mathematics and Statistics, Nanjing University of Information Science and Technology, Nanjing 210044, China

E-mail address: wjliu@nuist.edu.cn 